\title{
Phase transitions in two-dimensional traffic-flow models
}

\author{
José A. Cuesta, Froilán C. Martínez, Juan M. Molera, and Angel Sánchez \\ Escuela Politécnica Superior, Universidad Carlos III de Madrid, Avda. Mediterráneo 20, E-28913 Leganés, Madrid, Spain
}

(Received 17 May 1993)

\begin{abstract}
We introduce two simple two-dimensional lattice models to study traffic flow in cities. We have found that a few basic elements give rise to the characteristic phase diagram of a first-order phase transition from a freely moving phase to a jammed state, with a critical point. The jammed phase presents new transitions corresponding to structural transformations of the jam. We discuss their relevance in the infinite-size limit.
\end{abstract}

PACS number(s): 05.70.Fh, 05.40.+j, 64.60.Cn, 89.40.+k

Car displacements through large cities, data exchange among processors in a massively parallel computer, or communications in computer networks, are examples of situations where avoiding undesirable traffic jams is extremely important [1]. It is therefore necessary to achieve a comprehensive understanding of the mechanisms leading to the nucleation, growth, and evolution of these traffic jams, as they can be responsible for decrease or even full suppression of flow between different parts of the system. To this end, models based on cellular automata (CA) seem to be a suitable tool to approach the problem because of both their nature and their computational efficiency. Some one-dimensional [1-5] and two-dimensional $[6,7]$ models have been proposed in the past to study different traffic flow problems. In the one-dimensional case, there is good agreement between CA [4] and fluiddynamical [8] results, as well as with data obtained from actual traffic in highways $[1,4]$; however, from a theoretical point of view a great deal of work is still needed, although there has been recently some progress [5] by taking into account short-range correlations in mean-field theory. As regards two-dimensional (2D) CA models, the only ones we are aware of are based upon the fundamental assumption that cars never turn $[6,7]$. In this Rapid Communication we show that allowing cars to turn (as in actual situations) brings along the appearance of a complex phase diagram.

Our model consists of the following basic ingredients. We have cars moving inside a town. The town is made of one-way perpendicular ( $L$ horizontal and $L$ vertical) streets arranged in a square lattice with periodic boundary conditions. The way of every street is fixed independently according to a certain rule that depends on the particular choice of the model (see below). Cars sit at the crossings, and they can move to one of their nearest neighbors (allowed by the direction of the streets) every time step. Two cars cannot be at the same crossing simultaneously. Each car is assigned a trend or preferred direction, ruled by a variable $w_{i}(\mathbf{r})$, which we define as the probability that car $i$, located at node $\mathbf{r}$, jumps to the allowed neighbor in the horizontal street [accordingly, $1-w_{i}(\mathbf{r})$ is the probability to move vertically]. Finally, there are traffic lights that permit horizontal motion at even time steps and vertical motion at odd time steps.

We now define the dynamics of the model. Every time step $w_{i}(\mathbf{r})$ is evaluated and the direction where to move next is chosen accordingly. Then, it is checked that the chosen site is empty and that the motion is allowed by the light; otherwise the car will not be moved. Finally, all cars that can be moved are placed at their destination site and the next time step starts. We want to stress that the whole process is carried out simultaneously for all cars. The fact that traffic lights allow motion alternatively in vertical and horizontal streets prevents two cars from colliding at any crossing.

In this paper, we concern ourselves with two of the simplest versions of the model, which we name model A and model B. Both models are characterized by a unique parameter $\gamma$ which we call randomness. This parameter allows us to control the trend of the motion of every car by defining $w_{i}(\mathbf{r})$ through it. Model A has streets pointing only up and left. Half of the cars are given a trend $w_{i}(\mathbf{r})=\gamma$, and the other half are set to $w_{i}(\mathbf{r})=$ $1-\gamma$. This amounts to having half of the cars moving preferentially upwards and the other half leftwards. For symmetry reasons, it is enough to study the range $0 \leq$ $\gamma \leq 1 / 2$. It has to be noticed that, in case we fix $\gamma=0$, the cars are deterministic and always move along their preferred direction, and Model A becomes Model I of Ref. [6]. Model B is defined in the following way. It has streets that point alternatively up and down, and right and left. We work with four equal-number groups of cars: Each of them is assigned one of the four possible directions as its trend. This is accomplished by the following definition: Upward or downward bound cars have $w_{i}(\mathbf{r})=\gamma$ if a street with the same direction as the trend of the $i$ th car passes through site $\mathbf{r}$, and $w_{i}(\mathbf{r})=1-\gamma$ otherwise; left or right cars behave the other way around.

On the above described models, we have carried out an extensive simulation program, simulating the corresponding CA's on towns of $32 \times 32,64 \times 64$, and $128 \times 128$ sites. A typical run consists of the evolution along $10^{6}$ time steps of a randomly chosen initial condition for a given density (ratio of number of cars to number of lattice sites). For every time step we monitor the mean velocity, defined as the number of moved cars divided by the total number of cars. By means of this magnitude, we distinguish when the system reaches a steady state. Once in this state, we perform time averages on magnitudes of interest until the end of the simulation. We have also studied the outcome of different randomly chosen initial configurations. Although, in general, these out- 
comes are similar, a very particular dependence is found in some parts of the phase diagram (see below). We have also checked different random-number generators [9] and all of them lead to the same results. We have studied these models for a number of car densities ranging from $n=0$ to $n=1$, and for randomness values on the range $0 \leq \gamma \leq 1 / 2$. In addition, we have recorded any possible structure of the traffic jam in the steady state by measuring the average occupation time per site, defined as the number of time steps during which a site is occupied by a stopped car divided by the total averaging time. All simulations were performed in computer workstations HP 720, and DEC 3100 and 5100; a typical simulation for a given car density on a $64 \times 64$ town takes about 2 $\mathrm{h}$ of CPU time, and $12 \mathrm{~h}$ for a $128 \times 128$ town (this is for model $\mathrm{A}$; for model $\mathrm{B}$ times are approximately $25 \%$ higher).

Results for model A are summarized in Fig. 1. Such a figure can be understood as the phase diagram of a first-order phase transition [11] from a freely moving to a jammed phase. The curves $v(n)$ undergo a discontinuous transition of magnitude $\Delta v(\gamma)$ at density $n_{t}(\dot{\gamma})$. As $\gamma$ increases, $n_{t}(\gamma)$ shifts to higher densities and $\Delta v(\gamma)$ decreases, eventually vanishing for some randomness $\gamma_{c}$. The point of density $n_{c}=n_{t}\left(\gamma_{c}\right)$ and average velocity $v_{c}=v\left(n_{c}\right)$, belonging to the curve for $\gamma_{c}$, will correspond to a critical point. This conclusion is further supported by the large increase of the fluctuations of $v$ observed in the vicinity of that point. As can be inferred from Fig. 1, the location of the critical point lies somewhere in the range $0.45 \leq \gamma_{c} \leq 0.5$, but it cannot be more accurately determined from our simulations, first, because $\gamma$ is an input parameter, and, second, due to the strong size dependence of $\gamma_{c}$.

The part of the curves $v(n)$ corresponding to the free

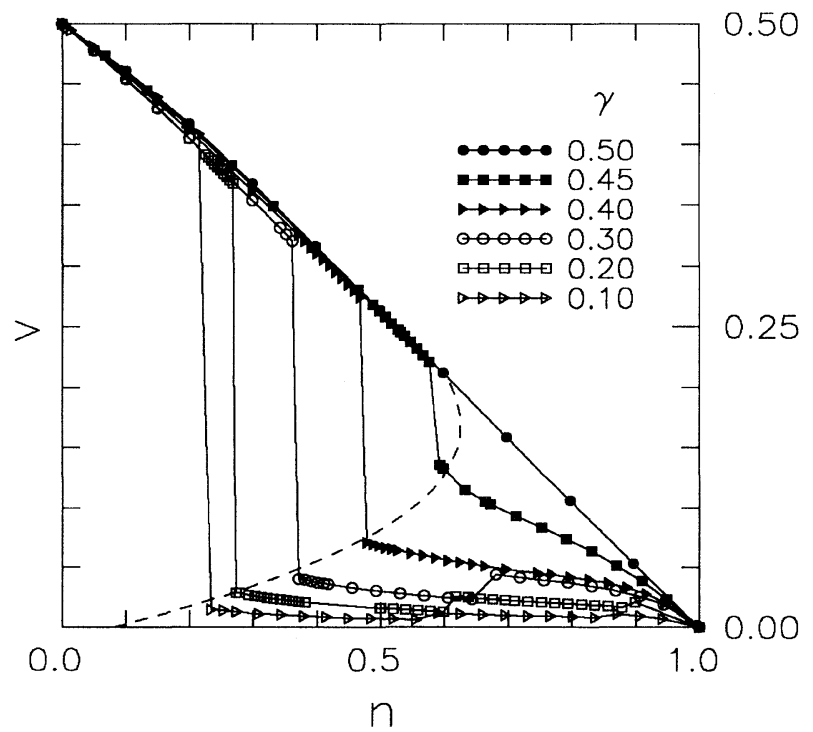

FIG. 1. Average velocity, $v$, for cars in model A as a function of car density, $n$, for different randomness values, $\gamma$, in a city of $64 \times 64$ streets. The full lines are a guide to the eye. The dashed line is an approximate fit to the points where the transition occurs. phase (which for $\gamma=1 / 2$ means the whole curve) fits rather well the linear law $v(n)=(1-n) / 2$. It can be proven analytically for the infinite system [10] that this is precisely the asymptotic behavior of $v(n)$ when $n \rightarrow 0$, for any value of $\gamma$. It is remarkable that the agreement with the simulations is rather good even far from this limit.

The jammed phase, and in fact the nature of the transition, can be better understood by analyzing the average distribution of cars on the lattice. Figure 1 shows in this phase, for the lowest values of $\gamma$, a few small jumps in which the value of $v$ increases. The explanation of these jumps is the following: Before the jamming transition occurs, cars are homogeneously distributed, whereas after the transition cars always ordered along broad diagonal strips extending throughout the whole system (see Fig. 2 ), with the two types of cars roughly separated in two halves. These strips do not trap empty sites (holes) inside; thus, the observed remnant average velocity is due only to the movement of the cars on the borders of the strips. Different numbers of strips characterize different ordered phases. A given initial configuration goes to one of these phases with a certain probability. For a given density, we compute this probability by taking a large number of initial configurations and counting how many of them go to each phase. The stable phase will be that of maximum probability, the rest of them being metastable. Accordingly, in Fig. 1 we plot the velocity of the stable phase. The small jumps correspond to an exchange of stability between two phases. In these jumps $v$ increases, since every new strip provides two more borderlines along which cars can move. As this remnant movement is just a "surface" effect, it should vanish when $L \rightarrow \infty$; however, at the same time the number of strips increases, hence

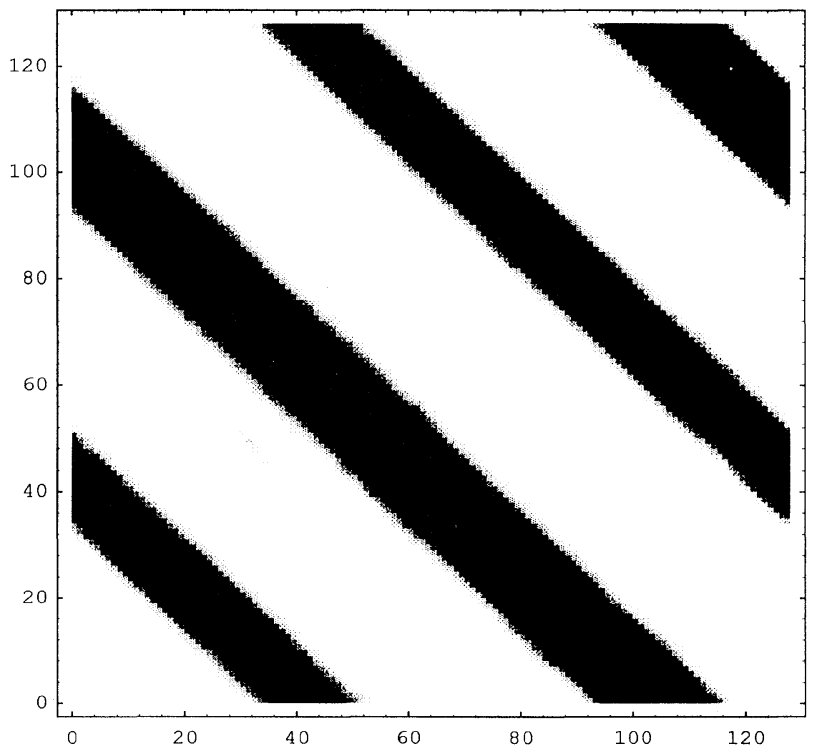

FIG. 2. Average site density of stopped cars in model A $(128 \times 128$ streets $)$ in the jammed phase, for a car density $n=0.7$ and a randomness $\gamma=0.1$. Different values are represented by different gray levels ranging from black (site always empty) to white (site always occupied). This illustrates the multistrip structure of the jammed phase in model A. 
supplying extra moving cars. The resulting value in the infinite system will depend on this competition of effects; we will return to this point later on.

For $\gamma=0$ the results are similar to those reported in [6] (the only difference being that our velocities are, by definition, half theirs). Since cars do not change direction, any initial configuration ends up either in a periodic or in a stuck $(v=0)$ state. The predominance of each kind of state decides in which phase the system is. According to the authors of [6], their results do not allow them to exclude the possibility that $n_{t}(0) \rightarrow 0$ as $L \rightarrow \infty$. In contrast, though in our simulations the values of $n_{t}(\gamma)$ also decrease as $L$ increases, we can clearly see that for the lowest values of $\gamma$ (say, $\gamma=0.1,0.2$, and 0.3 ) it already converges to a nonzero value, even for the relatively small sizes we are dealing with. Besides, as we have commented on above, we have proven [10] that the slope of $v(n)$, for infinite $L$, is exactly $-1 / 2$, independently of $\gamma$, in the limit $n \rightarrow 0$. This result also holds for $\gamma=0$; however, the simulations of [6] indicate that the value of the slope in the free phase is 0 for $L$ up to 512 . This fact supports the idea that when $L \rightarrow \infty, n_{t}(0) \rightarrow 0$, though the possibility of a change of slope from 0 to $-1 / 2$ at much larger system sizes is not excluded, but seems very unlikely.

From the size dependence of the parameters of our simulation we can draw an image of what happens in model A when $L \rightarrow \infty$. On the one hand, as we have already pointed out, $n_{t}(\gamma)$ converges to a nonzero value in the infinite system. On the other hand, the $\gamma=0.5$ curve does not change with $L$, and the critical point moves towards this curve as $L$ increases (it cannot be inferred from our results whether or not $\gamma_{c}$ finally reaches the value $1 / 2$ ). Accordingly, even though the transition densities, $n_{t}(\gamma)$, for the rest of the values of $\gamma$ still decrease, they should reach a nonzero value when $L \rightarrow \infty$. This part of the phase diagram will thus not qualitatively change at infinite size. Regarding the structure of the jammed phase, as strips never trap holes, the only possible way that such structures survive in an infinite city with $n \neq 0$ and $n \neq 1$ is that an infinite number of strips appear. Consequently, those "transitions" between jammed phases with different numbers of strips are a finite system size effect; a result that is further supported by the fact that such transitions move quickly towards $n_{t}(\gamma)$ as $L$ increases. The infinite system will then be formed by infinite strips with a typical size and a typical separation (which will in general depend on $n$ ), and the value of $v$ will simply be the ratio of the average number of moving cars per strip to the average number of cars per strip. Nevertheless, as the average separation between strips seems comparable to the sizes used in our simulations, the values of $v$ obtained are still affected by strong finite-size effects.

The phase diagram of model $B$ is similar to that of model A. There is also a phase transition from the "free" to the jammed regime, with diagonal strip structure right after the transition. This is indicated as before by a sharp decrease of the velocity for a certain value of the density $n_{t}(\gamma)$ (smaller than in model A). The dependence of the parameters characterizing the transition, $n_{t}(\gamma)$ and $\Delta v(\gamma)$, on $\gamma$ and the size of the city $L$ is qualitatively the same as in model A. The main differences of this model are in the structure of the jammed states appearing after the transition. This model having four different types of cars and streets, it has a symmetry (absent in model A) under $90^{\circ}$ rotations, and the jammed strip can appear with equal probability along each diagonal direction. Besides that, the strip has inside some holes that allow the diffusion through the jam of the different car types. They also contribute to the remnant velocity in the jam state and could have some significant effect in the infinite size case. The other important departure from the behavior of model $\mathrm{A}$ is the type of stable phases present in the jammed region. First of all, while in model $A$ the jammed phase can show multiple strips, in model $B$ we only see one strip. This strip is composed of two longitudinal halves, each containing a mixture of two types of cars. For example, if the strip runs from the lower left to the upper right, the upper half of it is mainly composed of cars of the types trying to go to the right and down, and the bottom half by the ones trying to go to the left and up. Second, as density is increased, a point is reached where the form of the stable jammed phase suddenly changes. The majority of the holes that before this point were forming a strip parallel to the cars now arrange themselves in a closed squarelike region (see Fig. 3). Meanwhile, the cars in the jam have separated in four regions according to their type. The cars trying to go up are above each empty region, to the left are the ones trying to go left, and so on. This change of structure produces a noticeable, though small, change in the slope of the velocity curves $v(n)$ in the phase diagram. Our simulations do not allow us to conclude whether this transition is continuous or weakly first order. More work on this point is in progress [10].

From our data we can only conjecture what the struc-

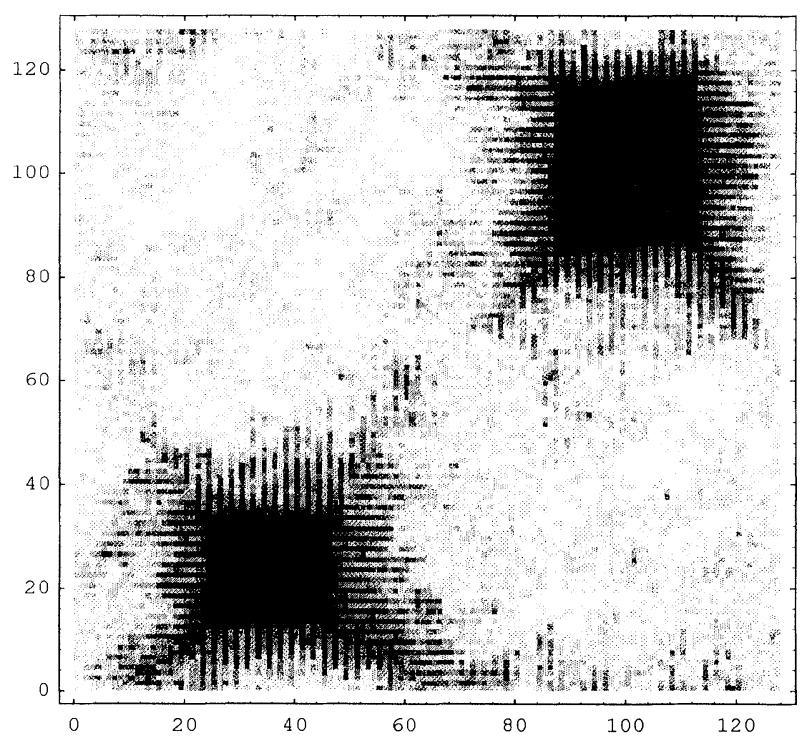

FIG. 3. Same as Fig. 2 for model B and car density $n=0.9$ and randomness $\gamma=0.2$. These parameters correspond to the second-order phase (see text). The existence of closed empty regions surrounded by cars is illustrated. 
ture of the jammed phase in model $\mathrm{B}$ will be as the size of system goes to infinity. We have already seen, in some preliminary runs, that the number of those empty regions increases, as we simulate, at constant density, in larger cities. Keeping the parallelism with model A, we can think that in the limit of infinite size an infinite number of strips will appear (though the existence of holes inside them weakens this conclusion). However, due to the $90^{\circ}$-rotation symmetry present in model $\mathrm{B}$, the strips may appear in both diagonal directions simultaneously. If this were to happen the stable state would be one in which there would be an infinite number of squarelike empty regions, as described above, arranged in a kind of lattice structure. We hope that we will be able to settle this question in the future.

In summary, we have studied models incorporating what we think are the essential ingredients (excluded volume and turn capability) of urban car movement in cities with realistic structures (defined by the arrangement of the streets and the organization of traffic lights) and we have found a first-order phase transition from a freely moving regime to a jammed state. It is important to check whether this striking feature still stands when more elements are added (say, disorder via forbidden streets or nonsynchronized traffic lights, preferred streets, rush hours, stopped cars [7], etc.). If this happens, it will be possible to conclude that, as a consequence of car interaction, any city will always have a saturation density of cars after which the average velocity falls sharply. This could be an important issue to consider in the design of city and traffic policies. If a city, with a given density of cars, were saturated, local improvements would have little effect on the average velocity, and only global changes in the city capacity or the number of cars would be able to solve the problem. More analytical and numerical work is currently being developed [10] along this line.

Finally, we want to point out that this work, as an example of nonequilibrium phase transitions, poses some very important questions regarding the universality of this kind of behavior and its appearance in other physical contexts. We envisage that models of crystal growth and ballistic deposition [7], surface roughening [5], granular flows [12], colloids, and, in general, systems where excluded volumes play an important role would be good candidates for research in this direction, as they incorporate the two main features of our models.

We acknowledge financial support of the Dirección General de Investigación Científica y Técnica (Spain) through two projects: PB92-0248 (F.C.M., J.M.M., and A.S.) and PB91-0378 (J.A.C.).

A 46, R6124 (1992)

[7] T. Nagatani, J. Phys. Soc. Jpn. 62, 1085 (1993).

[8] B. S. Kerner and P. Konhauser, in Europhysics Conference Abstracts 17A (13th General Conference of the Condensed Matter Division of the European Physical Society), edited by W. Heinicke (EPS, Geneve, 1993).

[9] W. H. Press, S. A. Teukolsky, B. P. Flannery, and W. T. Vetterling, Numerical Recipes in $C, 2$ nd ed. (Cambridge University, New York, 1992), Chap. 3.

[10] J. A. Cuesta, F. C. Martínez, J. M. Molera, and A. Sánchez (unpublished).

[11] H. E. Stanley, Introduction to Phase Transitions and Critical Phenomena (Oxford University Press, New York, 1971).

[12] J. Lee, HLRZ-KFA Jülich Report No. cond-mat/9306005 (unpublished). 


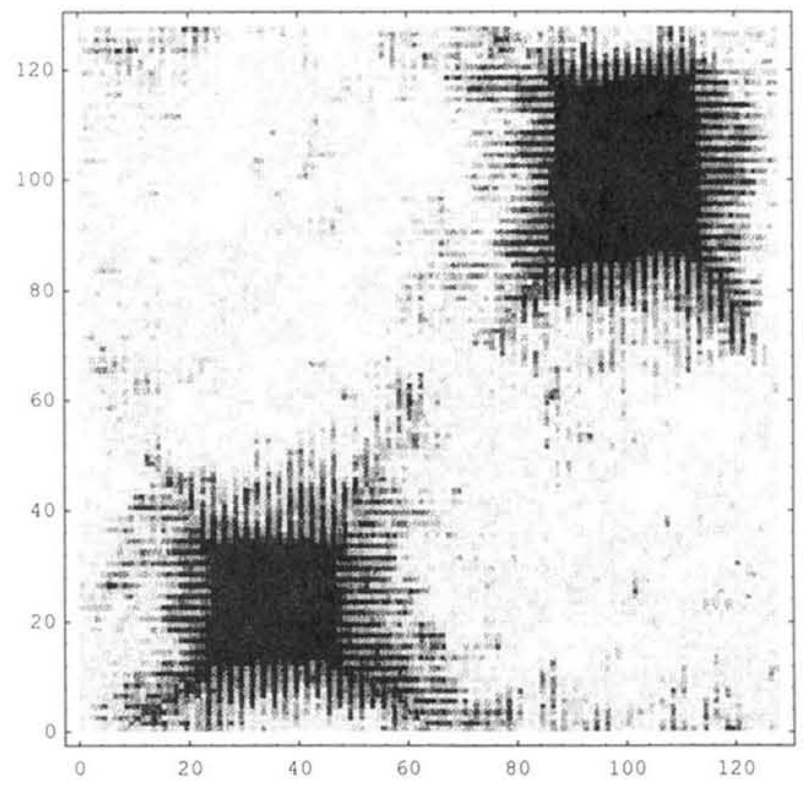

FIG. 3. Same as Fig. 2 for model B and car density $n=0.9$ and randomness $\gamma=0.2$. These parameters correspond to the second-order phase (see text). The existence of closed empty regions surrounded by cars is illustrated. 